\title{
Particle acceleration in solar flares: linking magnetic energy release with the acceleration process
}

\begin{abstract}
C. Dauphin
LUTh, Observatoire de Paris, 92195 Meudon Cedex, France

e-mail: cyril.dauphin@obspm.fr

Received 24 November 2006 / Accepted 21 May 2007

\section{ABSTRACT}

Context. Particles accelerated in solar flares draw their energy from the magnetic field. The dissipative scale of the magnetic energy in the solar corona implies that the magnetic energy is transmitted to the particles through a large number of dissipative regions (DR). Aims. To finally compute the particle energy distribution, we present an approach to linking the magnetic energy release to the acceleration process that occurs in each DR .

Methods. Although not directly observed, the magnetic energy release process is assumed to evolve in an SOC state that leads to the power-law behaviour of the probability distribution function for the energy released in each DR. We consider that most of the accelerated particles leave the acceleration region after one interaction, but we introduce a distribution of the particle acceleration lengths inside each acceleration region.

Results. We calculate the kinetic energy distribution of the accelerated particles. Our model allows us to identify the physical process that determines each part of the particle energy distribution.

Conclusions. As a conclusion, we discuss the limitations of the approach presented in this paper.
\end{abstract}

Key words. Sun: flares - acceleration of particles

\section{Introduction}

A solar flare is defined as a sudden transfer of magnetic energy to the ambient coronal plasma that leads to heating, flows, and particle acceleration. Observations and analyses of hard X-ray emissions are the most direct diagnostic of these non thermal particles. The last decades of observations have shown that a large number of particles are accelerated $\left(\sim 10^{38}\right.$ electrons s $\left.^{-1}\right)$ to energy going up to several $\mathrm{MeV}$, which implies a high efficiency for the acceleration mechanism.

One of the most relevant pieces of information that allows us to constrain the physical process that occurs during particle acceleration is certainly provided by the particle energy spectrum. Generally, hard X-ray spectra are reproduced by a simple or a double power-law electron spectrum (Holman et al. 2003; Conway et al. 2003; Piana et al. 2003). These electron spectra show a steep break generally between $10 \mathrm{keV}$ and $100 \mathrm{keV}$, and the spectral index below the break is usually lower than the spectral index at higher energy (Lin et al. 1982).

In this paper, an attempt is made to identify the physical processes that determines each part of the particle energy distribution. The basic idea is to take the particle acceleration process and the magnetic energy release process into account.

Particle acceleration mechanisms can be divided into three different classes: shock acceleration, turbulent acceleration, and direct electric field (further discussion can be found in the review of Miller et al. 1997). The energy of all aspects of the flare process, including particle acceleration, must ultimately come from the release of the stored magnetic energy. One possible way to release the magnetic energy stored in the corona is the formation of small-scale magnetic discontinuities where the frozen-in law is violated and where instabilities and anomalous resistivity can easily rise. Here, we consider that the particles are accelerated by the direct electric field that appears in such a region.

A large number of works have been devoted to analytical and numerical analyses of particle acceleration by a direct electric field in X-point (e.g. Hamilton et al. 2005), by a reconnecting current sheet (Litvinenko 1996; Zharkova \& Gordovskyy 2004; Wood \& Neukirch 2005), and in a spin and fan geometry (Litvinenko 2006; Dalla \& Browning 2005, 2006). These different studies usually found a power-law particle-energy distribution due to the configuration of the magnetic field that generates a chaotic behaviour of the particle trajectories.

The complex magnetic environment of an active region (Arzner \& Vlahos 2006), as well as the turbulent nature of photospheric motions that externally and continuously drives the system, will add new stresses to the existing large-scale topologies and lead to the formation of short-lived, small-scale magnetic discontinuities (see e.g., Vlahos et al. 2004; Fragos et al. 2004). Moreover, the properties of coronal turbulence implies that the magnetic energy is dissipated and transmitted to the particles on very small lengthscales (Einaudi \& Velli 1994). Thus, every model of particle acceleration during a solar flare has to take into account the large-scale physical process that leads to the appearance of several dissipative regions where the particle acceleration process can takes place.

The idea of multiple dissipation sites in flares has been modelled by Lu \& Hamilton (1991) by using the self-organised criticality (SOC) concept. Evolution of this model followed by satisfying the Maxwell's equations (e.g. Vlahos et al. 1995; Vassiliadis et al. 1998; Isliker et al. 2000). These SOC and associated cellular automata models identify dissipation occurring in many spatially separated regions. Several authors have investigated the problem of particle acceleration by using a model 
in which the accelerated particles move between the dissipation regions (Vlahos et al. 2004; Anastasiadis et al. 2004; Dauphin et al. 2007). Particles gain or lose energy in each dissipative region. The rate of energy change is proportional to the electric field, which is a function of the magnetic energy.

Another approach is to calculate the acceleration of particles in an electromagnetic field taken from MHD simulation or random-phase turbulence proxies or p-model (Decamp \& Malara 2006). To study particle acceleration, the electromagnetic field is frozen in time, and test particles are injected into the simulation box. The energy gain of the test particles is calculated by solving the relativistic equations of motion. Such an approach has been used by Dmitruk et al. $(2004,2005)$ in direct simulations of homogeneous 3D MHD turbulence and by Arzner \& Vlahos (2004) and Arzner et al. (2006), who have worked with random-phase turbulence proxies. Recently Onofri et al. (2006) addressed the problem of stochastic acceleration in turbulent electric field generated by $3 \mathrm{D}$ reconnection and Turkmani et al. $(2005,2006)$ addressed the issue of particle acceleration in a 3D coronal MHD model. Some particles undergo rapid and systematic energy gain and leave the computational box with relastivistic energy, whereas other particles do not intereact with the current sheets and gain no energy or little.

These different studies cannot simultaneously take into account the process that leads to the appearance of the acceleration regions and the particle acceleration process inside each of these regions. In this paper, we consider these processes by introducing two probability distribution functions that represent the spatial distribution of acceleration regions and the distribution of the particle acceleration lengths inside each acceleration region.

\section{Model}

\subsection{Particle energy distribution from one acceleration region}

We consider an isolated acceleration region (AR). Particles are accelerated by the electric field into the AR and gain energy from the magnetic energy. We equate the magnetic energy flux to the particle energy flux in order to determine the electric field into the acceleration region. The magnetic energy flux is given by the equation:

$P_{\mathrm{mag}}=\frac{1}{\pi} v_{\mathrm{in}} B_{\mathrm{free}}^{2} S_{\mathrm{AR}}$,

where $v_{\text {in }}$ is the velocity of the plasma entering into the AR, $B_{\text {free }}^{2}$ is the magnetic free energy, and $S_{\mathrm{AR}}$ is the surface of the AR. The particle energy flux is proportional to the electric field $E_{\mathrm{AR}}$ into the $\mathrm{AR}$ and to the average of the electron acceleration length $\Delta l_{\mathrm{e}}$ and proton acceleration length $\Delta l_{\mathrm{p}}$ over the total number of electrons and protons accelerated. Thus, the particle energy flux can be written as

$P_{\mathrm{par}}=\dot{N}_{\mathrm{e}} e E_{\mathrm{AR}}\left\langle\Delta l_{\mathrm{e}}\right\rangle_{n_{\mathrm{e}}}+\dot{N}_{\mathrm{p}} e E_{\mathrm{AR}}\left\langle\Delta l_{\mathrm{p}}\right\rangle_{n_{\mathrm{p}}}$,

where the number of particle entering into the AR per unit of time can be expressed by

$\dot{N}_{\mathrm{e}, \mathrm{p}}=4 S_{\mathrm{AR}} v_{\mathrm{in}} n_{\mathrm{e}, \mathrm{p}}$,

where $n$ is the particle density for protons and electrons. In this expression, we neglect the flux and energy gain of ions heavier than protons since they are less abundant than protons. Equating
Eqs. (1) and (2) with the assumption $P_{\mathrm{mag}}=\alpha P_{\mathrm{par}}$, we find the expression of the electric field,

$E_{\mathrm{AR}}=\alpha \frac{B^{2}}{4 \pi e\left(\left\langle\Delta l_{\mathrm{e}}\right\rangle_{n_{\mathrm{e}}} n_{\mathrm{e}}+\left\langle\Delta l_{\mathrm{p}}\right\rangle_{n_{\mathrm{p}}} n_{\mathrm{p}}\right)}$,

where $\alpha$, taken randomly between 0 and 1 , represents the amount of magnetic energy transferred to the particles. This expression allows us to determine the energy gained for one particle (electron or proton) by the expression

$\varepsilon_{\mathrm{e}, \mathrm{p}}=e E_{\mathrm{AR}}\left\langle\Delta l_{\mathrm{e}, \mathrm{p}}\right\rangle P(\Delta l)$,

where $\Delta l$ is the acceleration length for one particle, index e or $\mathrm{p}$ represents the electron or proton energy gained, and $P(\Delta l)$ is the probability distribution function of the particle acceleration length. By inserting Eq. (4), we obtain the electron energy gained:

$\varepsilon_{\mathrm{e}}=\alpha \frac{B_{\text {free }}^{2}}{2 \pi n(1+\beta)} P(\Delta l)$.

Here $n=n_{\mathrm{e}} / 2=n_{\mathrm{p}} / 2$ is the plasma density, and $\beta$ is defined as the ratio of the average acceleration lenghts $\frac{\left\langle\Delta l_{\mathrm{p}}\right\rangle_{\mathrm{p}}}{\left\langle\Delta l_{\mathrm{e}}\right\rangle_{\mathrm{e}}}$. The complete determination of the particle energy gained requires knowing $\beta$ and $P(\Delta l)$. Below, we assume $\beta=1$ for the salve of simplicity and thus $\varepsilon_{\mathrm{e}}=\varepsilon_{\mathrm{p}}=\varepsilon$.

We assume that the probability distribution function of the acceleration length is given by

$P(\Delta l)=k_{1}(\Delta l)^{-\delta}$,

where $k_{1}$ is constant and $\delta$ the spectral index of the power law distribution. The particle energy distribution is given by

$N(\varepsilon)=n P(\Delta l) \frac{\mathrm{d}(\Delta l)}{\mathrm{d} \varepsilon}$

By inserting the different expressions, we find

$N(\varepsilon)=k_{1} n\left(e E_{\mathrm{AR}}\right)^{\delta-1} \varepsilon^{-\delta}$

for the particle energy distribution. For one active region, the spectral index of the particle energy distribution is the one for the particle acceleration length distribution.

\subsection{Particle energy distribution in a "gas" of acceleration regions}

We now consider several DR leading to a "gas" of acceleration regions with different values of magnetic energy incoming into each AR. Figure 1 shows a schematic view of a global acceleration region (GAR) composed of multiple acceleration regions. Particles (electrons and ions) are accelerated in a different direction into each AR. Thus, electrons and ions are accelerated in the same direction outside of the global acceleration region. The total particle energy distribution outside of the global acceleration region corresponds to the sum of the particle energy distribution in each AR. In other words, every particle interacts only with one acceleration region.

We consider that the probability distribution function of the magnetic energy entering into the different AR is given by a power law (Vlahos et al. 2004, and references therein):

$P\left(B_{\text {free }}^{2}\right)=k_{2}\left(B_{\text {free }}^{2}\right)^{-\zeta}$. 


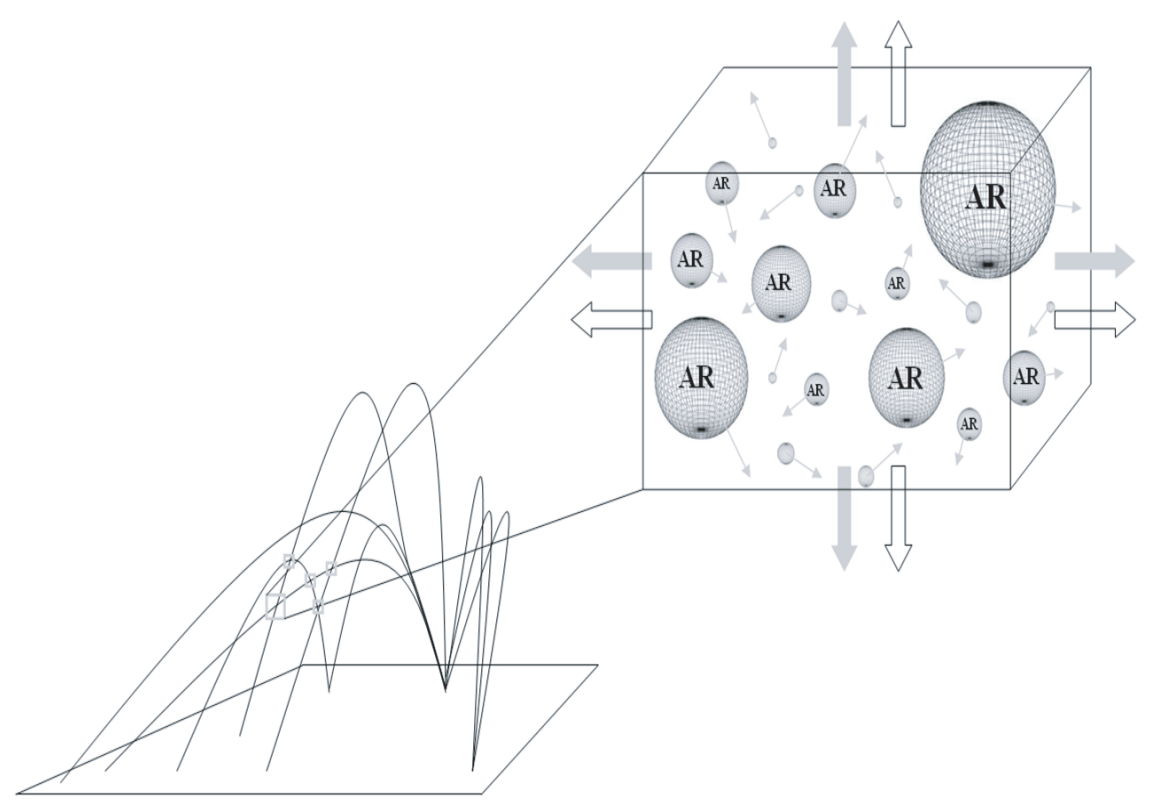

Fig. 1. Schematic view of a global acceleration region composed of multiple acceleration regions. The size of one AR is proportional to the value of the magnetic energy coming into the acceleration region. The small grey arrows represent the direction of accelerated electrons in each AR. The grey and white arrows outside the global acceleration region represent, respectively, the accelerated electrons and ions.

As mentioned in the introduction, such a distribution can result from the evolution of the magnetic field in a SOC state. Thus, the electric field distribution is given by:

$P\left(E_{\mathrm{AR}}\right)=k_{3}\left(E_{\mathrm{AR}}\right)^{-\zeta}$,

where $k_{2}$ and $k_{3}$ are constants. The total particle energy distribution can be written as

$N(\varepsilon)_{\text {total }}=\sum_{i=1}^{n_{\mathrm{AR}}} N(\varepsilon)_{\mathrm{AR}, i}$,

where $n_{\mathrm{AR}}$ is the total number of $\mathrm{AR}$ and $N(\varepsilon)$ is given by Eq. (9).

\section{Results}

We compute the total particle energy distribution given by Eq. (12) for different values of $n_{\mathrm{AR}}$. For each AR, we randomly select $\alpha$ between 0 and 1. Particles are injected into the acceleration volume with an initial Maxwellian distribution with a temperature of $10^{6} \mathrm{~K}$ and with initial velocities in the range $0<v<8 v_{\text {th }}$ where $v_{\text {th }}$ is the thermal velocity. The particle energy distribution for electrons and ions is calculated with a density of $n=10^{10} \mathrm{~cm}^{-3}$. We consider only super Dreicer events, thereby normalizing the minimal value of the electric field distribution to the Driecer electric field equal to $5.5 \times 10^{-4} \mathrm{~V} \mathrm{~cm}^{-1}$.

\subsection{Particle energy distribution in the case of one interaction for each particle}

We consider an example of particle acceleration from $10000 \mathrm{AR}$. The electric field value of each AR is simulated between $E_{D}$ and $10^{5} E_{D}$ with a spectral index equal to 1.6 (Vlahos et al. 1995). As an example, the particle's acceleration length values are included between $\sim 0.5 \mathrm{~km}$ and $\sim 16 \mathrm{~km}$ with a spectral index equal to 3 .

Figure 2 shows the particle energy distribution obtained from these 10000 AR. Several results can be drawn from this figure:

1. The distribution of the particle energy gain can be divided in three parts. The first one corresponds to the thermal

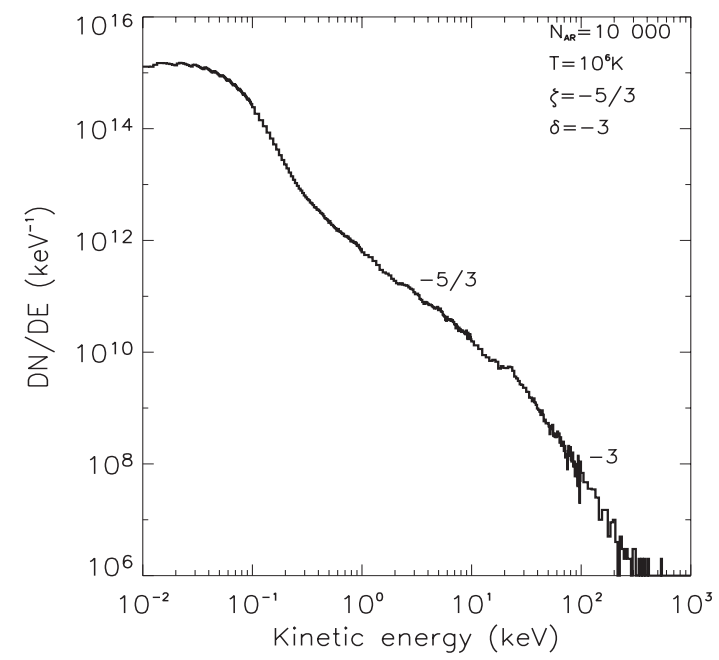

Fig. 2. Kinetic particle energy distribution obtained for $n_{\mathrm{AR}}=10000$, $\zeta=5 / 3$ and with a distribution of particle acceleration length given by a power law of spectral index $\delta=3$. The temperature of the injected Maxwellian is $10^{6} \mathrm{~K}$.

plasma injected with a temperature of $10^{6} \mathrm{~K}$ leading to a peak in the particle energy distribution at $43 \mathrm{eV}$. The second part of the spectrum is a power law of spectral index 1.6 corresponding to the spectral index of the magnetic energy release process. The last part of the spectrum is the particle energy distribution accelerated from the AR of the larger electric field. This part corresponds here to a power law of spectral index equal to 3 .

2. The break in the kinetic energy distribution occurs at $\varepsilon_{\text {break }}$, which corresponds to the energy gain of the particles of lower acceleration length in the AR with a higher electric field value. Thus, $\varepsilon_{\text {break }}$ is given by

$\varepsilon_{\text {break }}=e E_{\max } \Delta l_{\text {min }}$. 

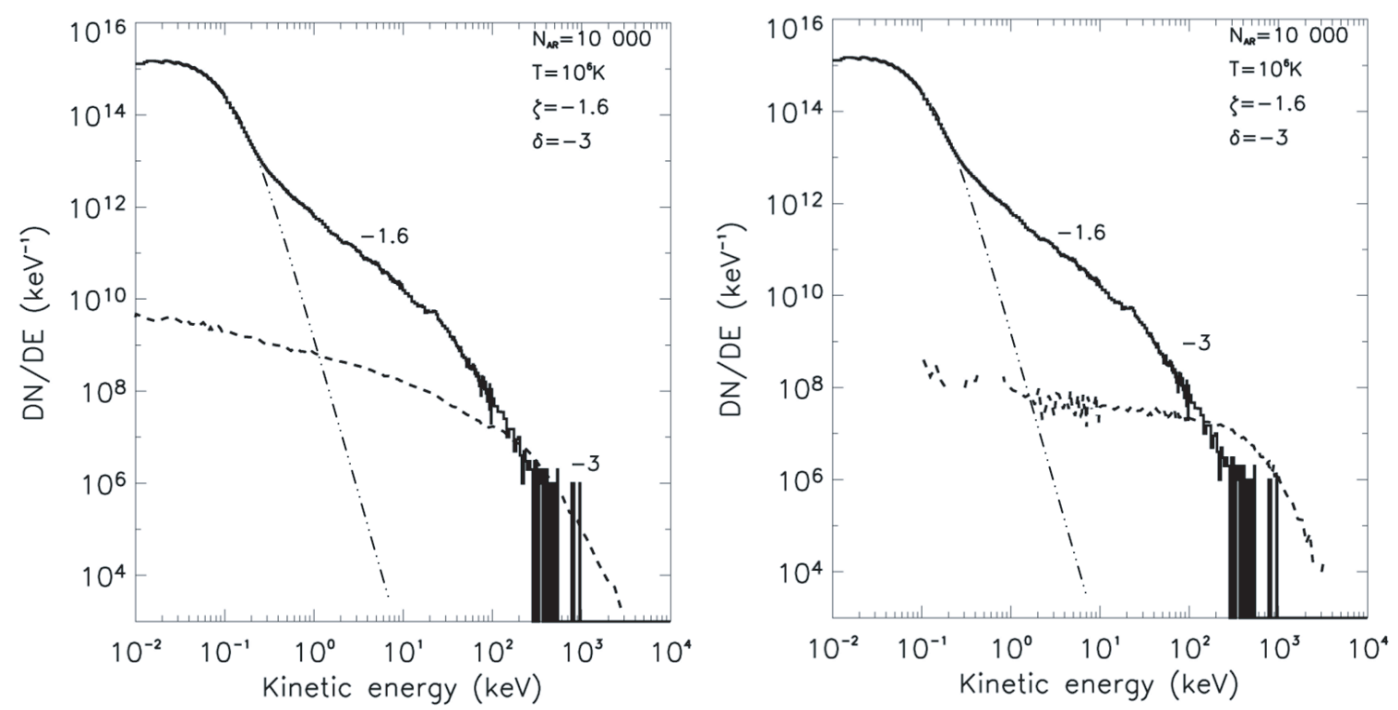

Fig. 3. Kinetic particle energy distributions obtained for $n_{\mathrm{AR}}=10000, \zeta=1.6$, and $\delta=3$. In the left panel, $10 \%$ of the total number of particles undergo between 1 and 1000 interactions (dashed line). In the right panel, 10\% of the total number of particles undergo between 1 and 10000 interactions (dashed line). The dash-dotted line represents the thermal part of the spectra.

In this case, the maximum value of electric field $\left(10^{5} E_{D}\right)$ is $55 \mathrm{~V} \mathrm{~cm}^{-1}$ and the minimum acceleration length is $500 \mathrm{~cm}$. The resulting energy break occurs at $27.5 \mathrm{keV}$. Moreover, the break in the kinetic energy distribution can be express as a function of the Alfven velocity. Indeed, $\varepsilon_{\text {break }}$ is proportional to $V_{\mathrm{A}_{\max }}^{2} \Delta l_{\min } /\langle\Delta l\rangle$.

The establishment of the double power law spectra is a function of the number of acceleration regions. For a low number of acceleration regions $\left(n_{\mathrm{AR}}=10\right)$, the probability of high electric field values is very low. Thus, particles are not efficiently accelerated, and the resulting particles energy distribution is the spectrum of the thermal plasma. By increasing the number of acceleration regions $\left(n_{\mathrm{AR}}=100\right)$, some of the acceleration regions have a large electric field and particles are then strongly accelerated outside the thermal part of the spectrum. This effect creates bumps in the spectrum at high energy. From $n_{\mathrm{AR}}=1000$, electric field values for the different AR are regularly distributed between $E_{\min }$ and $E_{\max }$. In this case, a double power law appears clearly outside the thermal part of the spectra.

Finally, we have to underline, in the case of one interaction for each particle, that the values of the spectral index below and above the break corresponds, respectively, to the spectral index value chosen for the probability distribution of the magnetic energy release and particle acceleration length.

The parameters $\beta$ and $P(\Delta l)$ determine the acceleration process of electrons and ions inside each acceleration region. Thus, the total ion energy distribution depends on the values of the two parameters. Although the exact determination of $\beta$ and $P(\Delta l)$ for electrons and ions is beyond the scope of this paper, we can discuss the effect of these two parameters on the ion energy distribution. The parameter $\beta$ models the possibility that electrons and ions are accelerated at different energies inside each acceleration region. Compared to the kinetic energy distribution of electrons, this parameter changes the value of the maximum energy gained by the ions and changes the value of the break in the ion energy distribution. The probability distribution function $P(\Delta l)$ changes the particle energy distribution above the break.

\subsection{Particle energy distribution in the case of several interactions for each particle}

We now consider that some of the accelerated particles undergo multiple interactions. Moreover, we consider that the interaction of an accelerated particle with an acceleration region leads to a loss or a gain in energy for the particle. Thus, the amount of energy transmitted to a given accelerated particle during its interaction with an acceleration region is given by

$\varepsilon_{\mathrm{e}}= \pm \alpha \frac{B_{\text {free }}^{2}}{2 \pi n} P(\Delta l)$

The plus or minus sign, selected randomly for each particle, represents the gain or the loss in particle energy.

Figure 3 shows the particle energy distribution obtained with $10 \%$ of the total number of particles that undergo several interactions. The distribution of the particle energy gain can be divided into four parts. The first one is the thermal plasma. The second part of the spectrum is due to the magnetic energy release process. The third part of the spectrum is due to the particle acceleration process in each acceleration region. The last part of the spectrum is created by the particles that undergo several interactions. Two comments can be drawn from this figure:

1. The spectrum of the particles that undergo multiple interactions wanders from a power law. With the increase in the number of interactions, each particle has a higher and equal probability of being accelerated by every value of the electric field. This results in a spectrum that is close to an inverse exponential function, since the particle trajectory is formally, equivalent to a Levy walk. In this case, the probability distribution function of energy gained at each interaction is given by a power law.

2. The increase in the number of interactions leads to the increase in the maximal energy gained by the particles.

Thus, particles accelerated by this stochastic process add a highenergy component in the particle energy distribution, which can leads to the formation of a new break at high energy. Such a break could explain the breaks observed at high energy in several flares. The value of the break mainly depends on the number of interaction. 


\section{Discussion and conclusions}

We have presented an acceleration model that assumes the acceleration of particles drawing their energy from the magnetic energy release in several dissipative regions. We consider that each dissipative region transmits a different amount of magnetic energy to the particles. Into each dissipative region, each particle has a different acceleration length that leads to a different energy gain. Thus, the small-scale physical processes that occur in acceleration regions are taken into account by introducing a distribution of the particles acceleration length inside each acceleration region.

In this model, the probability distribution function of the magnetic energy release is modelled by a power law. Furthermore, numerous acceleration models with various acceleration mechanisms (direct electric field in different magnetic field configurations) simulate the particle acceleration process in a single acceleration region (Dalla \& Browning 2005; Wood \& Neukirch 2004, 2005; Zharkova \& Gordovskyy 2004, 2005a). The results of these models support our assumption of a probability distribution function of the particle acceleration length given by a power law. Moreover, we consider that the probability function of the particle acceleration length and of the magnetic energy release are independent (i.e. the particle acceleraton length is independent of the magnitude of the electric field).

One of the new assumptions introduced in this paper is that most of the particles are accelerated one time. We use this model to compute the total particle energy distribution. Several results can be drawn from this distribution:

1. Outside of the thermal part, we can distinguish three parts in the particle energy distribution. The shape of the low-energy part is purely due to the magnetic energy release process. The high-energy component is due to the particles that undergo multiple interactions. Between these two parts, the particle energy distribution results from the particle acceleration process in each acceleration region. We can note that the values of the spectral index below and above the break are due to the choice of the free parameters of the model.

2. The energy of the break in the total particle energy distribution is given by the combination of the higher magnetic energy-release value with the minimum acceleration length.

3. The high-energy component in the particle energy distribution is due to the particles that undergo several interactions between the different acceleration regions. This high-energy component can lead to the formation of a new break at high energy. Such a break could explain the breaks observed at high energy in several flares.

In conclusion, we note that this model allows us to identify the physical processes that lead to the formation of the different parts of the particle energy distribution deduced from the observations. Such a particle acceleration mechanism can explain the main part of the energy distribution of the accelerated particles. However, other particle acceleration mechanisms, as acceleration by waves (Miller \& Roberts 1995), are certainly acting during a solar flare. The relative importance of each mechanism as a function of the particle energy has to be investigated further.

Acknowledgements. The author would like to thank L. Vlahos for helpful comments.

\section{References}

Anastasiadis, A., Gontikakis, C., Vilmer, N., \& Vlahos, L. 2004, A\&A, 422, 323 Arzner, K., \& Vlahos, L. 2004, ApJ, 605, 69

Arzner, K., \& Vlahos, L. 2006, A\&A, 454, 957

Conway, A. J., Brown, J. C., Eves, B., \& Kontar, E. 2003, A\&A, 407, 725

Dalla, S., \& Browning, P. 2005, A\&A, 436, 1103

Dalla, S., \& Browning, P. 2006, ApJ, 640, L99

Dauphin, C., Vilmer, N., \& Anastasiadis, A. 2007, A\&A, in press

Decamp, N., \& Malara, F. 2006, ApJ, 637, L61

Dmitruk, P., Matthaeus, W., Seenu, N., \& Brown, M. 2003, ApJ, 597, 81

Dmitruk, P., Matthaeus, W., \& Seenu, M. 2004, ApJ, 617, 667

Einaudi, G., Velli, M., Politano, H., \& Pouquet, A. 1996, ApJ, 457, 113

Fragos, T., Rantsiou, E., \& Vlahos, L. 2004, A\&A, 420, 719

Hamilton, B., Fletcher, L., McClements, K. G., \& Thyagaraja, A. 2005, ApJ, 625,496

Holman, G., Sui, L., Schwartz, R., \& Emslie, G. 2004, ApJ, 617, 667

Isliker, H., Anastasiadis, A., \& Vlahos, L. 2000, A\&A, 363, 1134

Litvinenko, Y. E. 1996, ApJ, 462, 997

Lu, E. T., \& Hamilton, R. J. 1991, ApJ, 380, 89

Lin, R. P., Mewaldt, R. A., \& Van Hollebeke, M. A. I. 1982, ApJ, 253, 949

Miller, A. J., \& Roberts, A. D. 1995, ApJ, 452, 912

Miller, A. J., Cargill, P. J., Emslie, A. G., et al. 1997, JGR, 102, A7, 14631

Onofri, M., Isliker, H., \& Vlahos, L. 2006, PhRvL, 96, 1102

Piana, M., Massone, A. M., Kontar, E. P., et al. 2003, ApJ, 595, 127

Turkmani, R., Vlahos, L., Galsgaard, K., et al. 2005, ApJ, 620, L59

Turkmani, R., Cargill, P. J., Galsgaard, K., et al. 2006, A\&A, 449, 749

Vassiliadis, D., Anastasiadis, A., Georgoulis, M., \& Vlahos, L. 1998, ApJ, 509, 53

Vlahos, L., Georgoulis, M., Kluiving, R., \& Paschos, P. 1995, A\&A, 299, 897

Vlahos, L., Isliker, H., \& Lepreti, F. 2004, ApJ, 608, 540

Wood, P., \& Neukirch, T. 2005, Sol. Phys., 226, 73

Zharkova, V. V., \& Gordovskyy, M. 2004, ApJ, 604, 884

Zharkova, V. V., \& Gordovskyy, M. 2005a, MNRAS, 356, 3, 1107

Zharkova, V. V., \& Gordovsky, M. 2005b, Space Sci. Rev., 121, 165 